\title{
EFECTO DE LA MUSICOTERAPIA EN EL ESTADO ANÍMICO Y CALIDAD DE VIDA DE UN GRUPO DE MUJERES SUPERVIVIENTES DE CÁNCER DE MAMA
}

\section{THE EFFECTIVENESS OF A MUSIC THERAPY TREATMENT ON MOOD AND QUALITY OF LIFE OF BREAST CANCER SURVIVORS}

\author{
Patricia Martí Augél,2, Melissa Mercadal-Brotons ${ }^{3}$ y Carme Solé Resano² \\ Fundació Oncolliga, Lliga Catalana d'Ajuda Oncològica \\ Universitat Ramon Llull. FPCEE Blanquerna. Barcelona \\ 3 Escola Superior de Música de Catalunya (ESMUC)
}

Resumen

Introducción: Las pacientes diagnosticadas de cáncer de mama pueden continuar experimentando malestar físico y emocional, pese a haber finalizado el tratamiento oncológico activo.

Objetivo: El objetivo de este estudio es explorar la eficacia de un tratamiento con musicoterapia para la mejora del estado anímico y de la calidad de vida de un grupo de mujeres supervivientes de cáncer de mama.

Método: Once mujeres con cáncer de mama en fase de supervivencia participaron en un programa de musicoterapia. El tratamiento ha consistido en 8 sesiones de musicoterapia de grupo, de frecuencia semanal y con una duración de 90 minutos/sesión. Las participantes completaron a nivel pre/post test la escala HADS (Hospital Anxiety and Depression Scale), la escala POMS-A (Profile of Mood States), el cuestionario de Calidad de Vida de la EORTCQLQ-30 (European Organization for Research and Treatment of Cancer-Quality of Life Core Questionnaire-30), así como una Escala de Valoración Numérica ( $E V N$ ) para el ítem Ansiedad, Tristeza y Malestar Físico a nivel de pre/ post sesión. También se administró un Cuestionario de Valoración al finalizar el programa.

Resultados: El tratamiento produjo mejora significativa en el índice de alteración emocional del POMS-A, así como en la subescala Tensión. No se observaron cambios significa-
Abstract

Introduction: Even when the oncologic treatments are completed, breast cancer patients still may experience physical and psychological distress.

Aim: The purpose of this study is to evaluate the effectiveness of a music therapy treatment on mood and quality of life of breast cancer survivors.

Method: Eleven free-disease women participated in a music therapy program. Treatment consisted of 8 weekly group music therapy sessions, 90 minutes/session. Participants completed the following instruments before and after the intervention (pre/post): HADS (Hospital Anxiety and Depression Scale), POMS-A (Profile of Mood States), Quality of Life EORTCQLQ-30 (European Organization for Research and Treatment of Cancer-Quality of Life Core Questionnaire-30). A 3 item Numerical Rating Scale (NRS) for Anxiety, Sadness and Physical Distress was administered at the beginning and ending of each session (pre/post session). An Evaluation Questionnaire was administered at the end of the program.

Results: The POMS-A results showed a statistical significant improvement in the Total Mood Disturbance Scores and in the Tension Subscale. No significant changes were found in the Anxiety and Depression HADS subscales. Quality of Life only significantly improved

Correspondencia:

Patricia Martí Augé

Fundació Oncolliga. C/ Rector Ubach, 5 - 08021 Barcelona

E-mail: patmarti.mł@gmail.com 
tivos en la variable Ansiedad y Depresión del HADS. La calidad de vida mostró un cambio significativo en la subescala Funcionamiento Emocional (EORTC-QLQ-30). Se ha obtenido una mejora significativa en los 3 ítems de la EVN. El Cuestionario de Valoración recoge un alto grado de utilidad y satisfacción de las participantes con esta modalidad de intervención.

Palabras clave: Cáncer de mama, supervivientes, musicoterapia, estado anímico, calidad de vida, tratamiento grupal. in the Emotional Functioning EORTC Subscale. A significant improvement has been found in the 3 NRS items. The Evaluation Questionnaire shows that participants have perceived this type of intervention as very useful and at a very satisfactory level.

Keywords: Breast cancer, survivor, music therapy, mood, quality of life, group treatment.

\section{INTRODUCCIÓN}

El cáncer de mama constituye actualmente un problema de salud. En España, es el tumor más frecuente en mujeres, y se diagnostican unos 22.000 casos nuevos al año ${ }^{(1)}$. Gracias a los programas de detección precoz y a los avances diagnósticos y terapéuticos, la tasa de supervivencia ha incrementado notablemente en los últimos 20 años, y se sitúa alrededor del 82,8\%. Este incremento de la supervivencia lleva hablar de este cáncer en términos de cronicidad $y$, muy frecuentemente, de cubrir necesidades y dificultades de tipo físico y psicosocial que no han quedado resueltas todavía. Problemas de adaptación a los cambios físicos, miedo a la recidiva, ansiedad, baja autoestima, aislamiento, malestar psicosocial,... son algunas de las dificultades emocionales más frecuentes que deben afrontar las personas supervivientes de cáncer ${ }^{(2,3)}$. La literatura señala un riesgo de morbilidad psiquiátrica de los supervivientes para la depresión y ansiedad, con una prevalencia que va del 0 al $58 \%$ para la depresión, y del 6 al 23\% para la ansie$\operatorname{dad}^{(4)}$. De ahí que recientemente se hayan ido desarrollando algunas iniciativas para abordar estas cuestiones, como el informe sobre las necesidades de los supervivientes de cáncer realizado por el Grupo Español de Pacientes con Cáncer ${ }^{(5)}$, el monográfico y plan de seguimiento para largos supervivientes de la Sociedad Española de Oncología Médica ${ }^{(6,7)}$ o también el Cuestionario CESC para medir necesidades no cubiertas del paciente superviviente de cáncer ${ }^{(8)}$.

Ser una paciente superviviente de cáncer de mama también puede significar que, aunque libre de enfermedad, la mujer siga experimentando la necesidad de hacer frente a una serie de dificultades. Así, las dificultades emocionales más frecuentes suelen ser "el miedo a la recidiva y/o a la muerte, sensación de pérdida de control, aislamiento, problemas de adaptación a los cambios físicos (pérdida de energía, cansancio, infertilidad, ganancia de peso, cambios en los patrones de sueño y alimentación, etc.), malestar psicosocial (ansiedad, depresión, baja autoestima y cambios en la imagen corporal), dificultades en las relaciones sexuales, redefinición o cambio de valores, cambios en las relaciones con las personas del entorno (familiares y amigos) y preocupaciones relacionadas con la actividad laboral“ (p.276) ${ }^{(9)}$.

En los últimos años se han ido llevando a cabo en nuestro país interesantes intervenciones psicológicas con este colectivo a fin de paliar algunas de las necesidades físicas, emocionales y sociales, disminuir los niveles de distrés emocional, facilitar la recuperación y adaptación a la vida cotidiana así como mejorar su calidad de 
vida $^{(9-12)}$. Paralelamente, se han empezado a desarrollar e implementar otras modalidades de intervención provenientes del campo de las terapias creativas como la musicoterapia, el arteterapia o la danza movimiento terapia ${ }^{(13-16)}$. En este estudio se pretende explorar la eficacia de un tratamiento de musicoterapia para mejorar algunas de estas necesidades de las mujeres supervivientes de cáncer de mama.

\section{Concepto de Musicoterapia}

La musicoterapia es la utilización profesional de la música y de sus elementos, aplicada como tratamiento en el contexto médico, educativo u otros, a fin de optimizar la calidad de vida, y mejorar la salud y el bienestar físico, social, comunicativo, emocional, intelectual y espiritual de las personas $^{(17)}$. Puede definirse también como "la utilización sistemática de la música, en un entorno terapéutico, dirigida por un musicoterapeuta profesional, para restaurar, mantener y/o mejorar la salud de las personas. La finalidad es promover cambios deseables en el funcionamiento físico, cognitivo y/o psicosocial de las personas que viven un problema de salud..."(18).

Las aplicaciones de la música en el contexto médico y sanitario han estado presentes desde épocas lejanas y el estudio y aplicación de la música como recurso terapéutico ha ido evolucionando en función de las creencias y costumbres de cada época ${ }^{(19,20)}$. Hoy en día, la musicoterapia es ya una disciplina científica. La aplicación de la musicoterapia en el campo médico ha ido experimentando un creciente interés desde los años '80 y como consecuencia de los resultados satisfactorios que se han ido obteniendo en diversos estudios e investigaciones ${ }^{(21,22)}$. El número de estudios científicos sobre los efectos de la música en el tratamiento médico continúa creciendo, y ofrece valiosas aportaciones a los profesionales del campo de la sa- lud, así como interesantes implicaciones para futuras investigaciones y aplicaciones clínicas. Esta disciplina ha mostrado sus efectos beneficiosos en diferentes campos de la salud, siendo el oncológico uno de ellos.

\section{La Musicoterapia en Oncología y Cáncer de Mama}

La aplicación y eficacia de la musicoterapia en el contexto oncológico ha sido estudiada y analizada durante los últimos 30 años. Así, la primera descripción de la aplicación de la musicoterapia en cáncer fue realizada por Munro y Mount ${ }^{(23)}$ en el año 1978. Desde entonces, diferentes estudios han ido contribuyendo al conocimiento y difusión del rol de la musicoterapia en este contexto, hecho que ha ayudado a crear un espacio y una función de la musicoterapia en el campo de la oncología y psicooncología. Autores como $\mathrm{O}^{\prime}$ Callaghan y Hiscock ${ }^{(24)}$ utilizan el término «Musicoterapia Oncológica» para referirse a intervenciones con musicoterapia dirigidas a enfermos oncológicos de todas las edades, y con diferentes pronósticos de cáncer, y en diferentes momentos de la enfermedad. Magill ${ }^{(25)}$ habla de «Musicoterapia Integrativa», una especialidad de la musicoterapia que se contempla dentro de los programas de la Oncología Integrativa, y que "es aplicada para tratar múltiples síntomas como el dolor, trastornos del estado anímico y aspectos de comunicación. La música puede mejorar el dolor y síntomas como el miedo, ansiedad, depresión, frustración y soledad. La musicoterapia reduce los efectos de los estímulos nocivos mientras que eleva el estado anímico y los sentimientos de confort y sensación de control" (p. 79).

De hecho, la literatura muestra cada vez más la importancia de las terapias no farmacológicas y complementarias para controlar el dolor y otros síntomas rela- 
cionados con enfermedades crónicas. Dos metanálisis realizados en el contexto de la musicoterapia en medicina, muestran resultados significativos para variables como náuseas, vómitos, estado anímico, dolor y bienestar $^{(21,22)}$. Diferentes programas han mostrado cómo la musicoterapia oncológica es un coadyuvante efectivo y una intervención terapéutica que -ofreciendo un enfoque multimodal e integral-permite cuidar las necesidades del paciente tanto a nivel fisiológico como psicosocial ${ }^{(26-34)}$. Los pacientes que participan en un tratamiento de musicoterapia suelen describir la experiencia como positiva, incluyendo una mejora en el estado anímico y bienestar, un aumento de la comunicación familiar, y un aumento de la esperanza y fuerza interior.

Aunque el número de publicaciones sobre musicoterapia en oncología continúa creciendo, pocas son las publicaciones que se pueden encontrar actualmente que vayan exclusivamente dirigidas al cáncer de mama, y todavía menos al cáncer de mama en fase de supervivencia. La mayoría de estudios localizados en la literatura incluyen tratamientos de musicoterapia dirigidos a muestras de pacientes diagnosticadas de cáncer de mama junto con pacientes con otros tipos de cáncer. Aún y así, determinados artículos sí muestran estudios e intervenciones diseñados para mujeres afectadas de cáncer de mama, y cómo la música puede ofrecer acompañamiento y apoyo durante todo el proceso oncológico a lo largo de las diferentes fases de la enfermedad.

En momentos previos al posible diagnóstico, la música puede ayudar a disminuir los niveles de ansiedad durante las mamografías $^{(35)} \mathrm{o}$ bien durante pruebas tan invasivas como las biopsias ${ }^{(36)}$. Durante la fase de tratamiento encontramos interesantes intervenciones de musicoterapia para ofrecer apoyo. Así, en el contexto quirúrgico, puede ofrecer soporte emocional y ayudar a controlar síntomas de malestar postoperatorio como el dolor ${ }^{(37-39)}$. También acompaña a pacientes oncológicos que reciben quimioterapia ${ }^{(40-47)}$, y hay autores que han estudiado la influencia de la música específicamente en mujeres con cáncer de mama en el momento en que están recibiendo la quimioterapia ${ }^{(48,49)}$. También hay algunos estudios que han empezado a valorar la eficacia de la música en pacientes que reciben radioterapia en el hospital( ${ }^{(50-52)}$. Parece ser que la musicoterapia ofrece interesantes beneficios a mujeres que se encuentran recibiendo terapia hormonal| ${ }^{(53)}$. Una de las grandes intervenciones de esta disciplina se da en el contexto y fase paliativa. Hay muchos estudios que analizan la influencia de la música en enfermos oncológicos que reciben cuidados paliativos ${ }^{(28,54-67)}$. En estos casos, se trata también mayoritariamente de estudios que incorporan sujetos con diferentes diagnósticos oncológicos y no están únicamente centrados en el cáncer de mama.

\section{La Musicoterapia en Mujeres Supervivientes del Cáncer de Mama}

Pocas son las publicaciones que hacen referencia a mujeres con cáncer de mama que se encuentran en fase de supervivencia de la enfermedad ${ }^{(14,16,68-70)}$. Hay otros estudios que también describen experiencias en esta fase, si bien en ellas participan personas con diferentes diagnósticos de cáncer, y no exclusivamente cáncer de mama $^{(71)}$ ni especifican cuánto tiempo ha transcurrido desde el fin del tratamiento activo. La finalidad principal de este estudio es evaluar la eficacia de una intervención de musicoterapia en el estado anímico y calidad de vida de un grupo de mujeres que han sido diagnosticadas de cáncer de mama y que se encuentran en fase de intervalo libre de enfermedad.

En situaciones de distrés emocional, la música permite modular el estado de 
ánimo y elevarlo. Como ya se ha expuesto anteriormente, algunas publicaciones han descrito los beneficios específicos de la musicoterapia en pacientes con cáncer de mama así como de otros tipos de cáncer en cuanto a la mejora del estado anímico ${ }^{(71-74)}$. En el contexto de la musicoterapia, éste es un objetivo terapéutico frecuentemente abordado: en un estudio desarrollado por Kruse ${ }^{(29)}$ para determinar las tendencias de la práctica clínica de la musicoterapia en el contexto oncológico en EEUU se observó que el 100\% de los musicoterapeutas entrevistados tenían como objetivo principal de intervención promover la expresión de sentimientos y emociones. El estado anímico es, pues, una interesante variable a seguir estudiando en el contexto del cáncer y concretamente durante esta fase de supervivencia.

Algunas de las técnicas aplicadas a las sesiones de musicoterapia, que pueden ayudar a elevar el estado de ánimo son: tocar instrumentos musicales, cantar, audiciones, y técnicas de relajación acompañadas de música, entre otras. Técnicas como estas permiten favorecer la expresión emocional, facilitar la focalización de la atención en la música (para distraer así de pensamientos negativos o preocupaciones), aumentar la interacción social y promover estados de relajación. Paralelamente, estas estrategias pueden ayudar a disminuir la ansiedad, la depresión y/o el malestar físico. El enfoque no verbal de la música, juntamente con el procesamiento verbal, puede ser de ayuda para las mujeres que han pasado por una experiencia de diagnóstico y tratamiento del cáncer de mama, ya que ofrece oportunidades para expresar aspectos relacionados con la enfermedad, para comunicarse con otras personas que están pasando por una situación similar a la suya, y para desarrollar e integrar en su día a día nuevos recursos de afrontamiento para esta nueva etapa $^{(14,16)}$.
Los programas diseñados en formato de grupo pueden ayudar a compensar los sentimientos de soledad y conductas de aislamiento, permitiendo la expresión personal y la reflexión conjunta, trabajando habilidades de comunicación y relación con los demás, promoviendo el apoyo mutuo, y contribuyendo así a mejorar el bienestar emocional. Es por esta razón que se ha diseñado una intervención en formato de grupo y aplicando las técnicas y estrategias de musicoterapia comentadas anteriormente.

Las intervenciones realizadas hasta ahora en el campo de la musicoterapia en el entorno médico y sanitario han sido mayoritariamente desarrolladas en países extranjeros. Aún y así, desde hace unos años, se están llevando a cabo en nuestro país interesantes proyectos y estudios de musicoterapia en este entorno $y$, muy especialmente también, en el contexto oncológico $^{(76)}$.

Los objetivos específicos de este estudio fueron:

1. Evaluar si hay una mejora del estado anímico y calidad de vida de un grupo de mujeres diagnosticadas de cáncer de mama que se encuentran en fase de supervivencia una vez finalizado el tratamiento de 8 sesiones de musicoterapia (pre/post tratamiento)

2. Evaluar si hay una mejora del estado de ánimo después de cada sesión de musicoterapia (pre/post sesión).

\section{MÉTODO}

\section{Muestra}

La muestra inicial estaba constituida por un total de 11 pacientes diagnosticados de cáncer de mama que se ajustaban a los siguientes criterios de inclusión: Mujeres adultas (mayores de 18 años), 
diagnosticadas de cáncer de mama, que han finalizado el tratamiento oncológico activo (cirugía, quimioterapia, radioterapia, excluyendo tratamiento hormonal adyuvante) al menos en los últimos 3 meses antes de iniciar la primera sesión de musicoterapia, que no hayan recibido previamente un tratamiento de musicoterapia y que hayan dado su consentimiento para participar en este estudio. Fueron excluidas del estudio aquellos sujetos que estaban en tratamiento oncológico activo (y por tanto, recibiendo cirugía, quimioterapia, radioterapia), que tenían antecedentes psiquiátricos graves o que presentaban problemas de hipoacusia moderada o severa. Con respecto a los sujetos participantes, un criterio de inclusión adicional fue el de haber asistido al menos al 50\% de las sesiones de musicoterapia. Así, de la muestra inicial, 3 sujetos fueron excluidos del análisis final por presentar un porcentaje inferior de asistencia. Por todo ello, la muestra final incluye un total de 8 sujetos $(\mathrm{N}=8)$, todas ellas mujeres, con un rango de edad de 41-76 años ( $M=56,75$, $\mathrm{DT}=12,71)$. Con respecto al estado civil, 3 estaban casadas, mientras que 3 eran viudas y 2 estaban solteras. Tres participantes tenían estudios básicos, 3 estudios secundarios y 2 superiores. La tabla 1 recoge los datos sociodemográficos y clínicos más relevantes de esta muestra.

\section{Diseño del proyecto}

Se trata de un diseño intrasujeto, de medidas repetidas. La variable independiente es la participación en un grupo de musicoterapia. Las variables dependientes a largo plazo (8 semanas, pre y post-tratamiento) son el estado de ánimo percibido por las participantes y evaluado mediante el POMS-A y el HADS así como la calidad de vida, evaluada mediante el EORTC-QLQ-30. Las variables dependientes a corto plazo (90 minutos de sesión, pre y post-sesión) incluyen cambios en ansiedad, depresión y malestar físico, evaluados a nivel pre- y post-sesión mediante una Escala de Valoración Numérica de 11 puntos (0-10).

\section{Instrumentos de evaluación}

Para evaluar los resultados a largo plazo (8 semanas) se utilizaron los siguientes instrumentos: Escala Hospitalaria de Ansiedad y Depresión (Hospital Anxiety and Depression Scale, HADS)(77), Escala Profile of Mood States (POMS-A) ${ }^{(78)}$, y el Cuestionario de Calidad de Vida de la European Organization for Research and Treatment of Cancer (EORTC-QLQ-30) ${ }^{(79)}$. Los participantes completaron estos instrumentos antes de iniciar la sesión 1, y después de haber finalizado la 8. Se utilizó una Escala de Valoración Numérica (EVN) para medir los resultados a corto plazo (90 minutos) en cuanto a ansiedad, depresión y malestar físico. Además, la musicoterapeuta utilizó una Hoja de Observación y Registro de Conductas (instrumento propio, elaborado ad hoc) después de cada sesión a fin de recoger las respuestas de cada participante así como un Cuestionario de Valoración del Programa de Musicoterapia (instrumento propio, elaborado ad hoc).

La Escala Hospitalaria de Ansiedad y Depresión (Hospital Anxiety and Depression Scale, HADS) ${ }^{(77)}$ es una escala ampliamente utilizada en oncología, que evalúa la presencia de síntomas de ansiedad y depresión en pacientes con enfermedad física en el contexto hospitalario. Tiene un total de 14 ítems, repartidos en dos subescalas de 7 ítems cada una, para medir: Ansiedad y Depresión. Consta de 4 opciones relativas a la frecuencia con la que se da un cierto tipo de respuesta definida como de ansiedad o de depresión, en un marco temporal de los últimos 7 días. Para este estudio se utilizó la versión española, adaptada de Caro e Ibáñez ${ }^{(80)}$. 


\section{Tabla 1. Características Sociodemográficas y Clínicas de la Muestra Final de Pacientes ( $N=8$ )}

\section{VARIABLES SOCIODEMOGRÁFICAS}

\section{Sexo}

Mujeres

$n=8$

Edad

41-76 años $(M=56,75 ; D T=12,71)$

\section{Estado civil}

Soltera

$n=2$

Casada

$n=3$

Viuda

$n=3$

$\begin{array}{ll}\text { Número de Hijos } & \\ \text { Sin hijos } & n=2 \\ 1 & n=3 \\ 2 & n=2 \\ 3 & n=1\end{array}$

Nivel de Estudios
Primarios
$n=3$
Secundarios
$n=3$
Superiores
$n=2$

Situación laboral actual
En activo
$n=3$
Baja laboral
$n=2$
Jubilada
$n=3$

\section{VARIABLES CLÍNICAS}

\section{Diagnóstico}

Cáncer de Mama

$$
n=8
$$

Período de tiempo transcurrido desde el fin del tratamiento activo

Entre 3 y 6 meses

Entre 7 y 12 meses

Entre 13 y 18 meses

Entre 19 y 24 meses

$$
\begin{aligned}
& n=1 \\
& n=1 \\
& n=5 \\
& n=1
\end{aligned}
$$

Tratamientos previos

Cirugía + Quimioterapia + Radioterapia $\quad n=7$

Cirugía + Radioterapia

$n=1$

\section{Tratamiento actual}

Terapia Hormonal

Ninguno

$n=5$

$n=3$

La Escala Profile Moods States (POMS)(78) consta de 58 ítems que permite evaluar el estado de ánimo. Para este estudio, con el objetivo de facilitar su aplicación a las pacientes, se utilizó la versión reducida (POMS-A) de 15 ítems elaborada por Fuen- 
tes, García-Merita, Melià y Balaguer ${ }^{(81)}$. Se trata de un auto-informe que incluye 4 escalas de afectos negativos, Tensión, Depresión, Cólera y Fatiga, y una escala de afecto positivo, Vigor. Consta de 15 ítems, que son adjetivos sobre diferentes estados afectivos de los que el paciente ha de graduar la intensidad de cada uno de ellos según los haya sentido durante los últimos 7 días, y en un formato de escala Likert de 5 puntos. También ha sido frecuentemente utilizada en el campo de la oncología así como de la musicoterapia.

El cuestionario de Calidad de Vida de la European Organization for Research and Treatment of Cancer-Quality of Life Core Questionnaire-30 (EORTC-QLQ-30), de Aaronson et al. ${ }^{(79)}$ es un instrumento para evaluar de manera específica la calidad de vida en sus diferentes dimensiones en pacientes con cáncer. Consta de 30 ítems, 28 de ellos con 4 alternativas de respuesta y 2 de ellos con 7 alternativas (donde 1 es pésima y 7 excelente), y el marco temporal se refiere a la semana previa. Está compuesto de 3 escalas: Estatus de Salud Global, Escalas Funcionales, y Escala de Síntomas. Para este estudio se ha utilizado la versión española de Arrarás, Illarramendi y Valerdi ${ }^{(82)}$, y se han utilizado la Escala Global y las 5 Escalas de Funcionamiento. Si bien existe un módulo específico que complementa el cuestionario y va dirigido a pacientes con cáncer de mama (EORTC-BR23) se desestimó su administración por una razón práctica, ya que en este estudio el test se rellena en el mismo momento antes de iniciar la sesión y requeriría alargar aún más el tiempo de administración de los test, cuando las dificultades de concentración todavía suelen ser un síntoma frecuente en pacientes que han terminado el oncológico activo, y por una razón de respeto a las pacientes, a fin de no resultar demasiado invasivos con preguntas y cuestiones de carácter muy íntimo. Es un test habitualmente utilizado en el campo de la musicoterapia.
La Escala de Valoración Numérica (EVN) es una escala empleada habitualmente en el contexto hospitalario como un instrumento rápido y sencillo de screening, que tiene como objetivo evaluar la intensidad de un síntoma. En este estudio se utilizó una ENV de 11 puntos (0-10), en la que se presenta una línea recta horizontal numerada de 0 a 10, en cuyos extremos se representa la polarización de la intensidad del síntoma (donde 0 es la ausencia del síntoma y 10 el síntoma a un nivel máximo e insoportable). Se han presentado 3 EVN para los 3 ítems elegidos: Ansiedad, Tristeza y Malestar Físico, y se pide a los participantes que las completen al inicio y final de cada sesión. Las EVN miden el cambio tras 90 minutos de haber participado una sesión de musicoterapia.

Para evaluar el estado anímico, además de estos tests estandarizados, se utilizó también una Hoja de Observación y Registro de Conductas (instrumento propio elaborado ad hoc) después de cada sesión a fin de recoger las respuestas/información de cada participante en cuanto a los siguientes aspectos: a) expresión emocional, b) atención-participación en la tarea musical, c) interacción-comunicación social, y d) asistencia a la sesión.

Una vez finalizado el programa, se pidió a las participantes que rellenaran también un Cuestionario de Valoración del Programa de Musicoterapia (instrumento propio, elaborado ad hoc), de manera anónima, para proporcionar feedback e información sobre sus experiencias en las sesiones de musicoterapia.

\section{Procedimiento}

El proyecto de este estudio fue inicialmente presentado al Comité de Patología Mamaria del Hospital del Mar - Parc de Salut Mar de Barcelona a fin de dar a conocer los objetivos y metodología del mismo. Una vez recibida su aprobación, se 
procedió a coordinar el proyecto con la enfermera gestora de casos de Cáncer de Mama, quien se ocupó de reclutar a las pacientes para este estudio. Aquellas que cumplían con los criterios de inclusión fueron derivadas al programa de tratamiento con musicoterapia. Las participantes leyeron una Hoja de Información del Estudio y firmaron el documento de Consentimiento Informado antes de empezar a participar en musicoterapia.

Antes de empezar el tratamiento (antes de la sesión 1), se procedió a la administración de los instrumentos de evaluación (HADS, POMS-A y EORTC-QLQ-30). Además, antes de iniciar cada una de las 8 sesiones y después de finalizar las mismas, se solicitaba a cada participante que reIlenase la Escala de Valoración Numérica para cada uno de los 3 ítems. Después de cada sesión, la musicoterapeuta cumplimentaba, para cada paciente, la Hoja de Observación y Registro de Conductas observadas en la sesión. Una vez finalizado el tratamiento, se volvieron a aplicar los mismos instrumentos de evaluación, juntamente con el Cuestionario de Valoración de la Experiencia de Musicoterapia.

Semanalmente, se implementaba una sesión de musicoterapia de grupo de 90 minutos de duración, en una de las salas del hospital adjudicada temporalmente para este proyecto.

\section{Sesiones de Musicoterapia}

Los objetivos terapéuticos del programa de musicoterapia iban dirigidos a cubrir el área emocional y social-interactiva a fin de mejorar el estado de ánimo de las participantes. Otros objetivos adicionales se dirigían a las áreas cognitiva y física. Todas las sesiones fueron dirigidas por la misma musicoterapeuta profesional, especializada a su vez en psicooncología. El programa de musicoterapia se basa en una orientación cognitivo-conductual principalmente, si bien recoge también algunos aspectos de otras orientaciones.

La intervención de musicoterapia consistió en la aplicación de diferentes técnicas y actividades musicales, siempre diseñadas e implementadas para trabajar los objetivos terapéuticos dirigidos a favorecer la expresión y ventilación emocional, canalizar emociones y sentimientos a nivel verbal y no verbal mediante la música, facilitar estados de relajación y distracción así como promover la interacción grupal. En el contexto oncológico, en el que las pacientes, pese a haber finalizado los tratamientos deben convivir con incertidumbre con respecto a su estado de salud, es recomendable ofrecer un espacio de musicoterapia estructurado, que aporte seguridad y predictibilidad. Es por esto que este programa sigue la misma estructura en cada sesión: se inicia con una actividad de apertura, se va desarrollando mediante la implementación de diferentes técnicas y actividades relacionadas con el tema a abordar en cada sesión, y finaliza con una actividad de cierre. Se combinaron técnicas de carácter activo (tales como cantar canciones, análisis lírico, tocar instrumentos musicales, improvisación instrumental, música y movimiento) con otras de tipo pasivo-receptivo (como son la relajación acompañada de música y las audiciones musicales).

Aspectos éticos

El proyecto de estudio recibió la aprobación del Comité de Patología Mamaria del Hospital del Mar - Parc de Salut Mar. Las pacientes que cumplían los criterios de inclusión fueron informadas del proyecto. Aquellas que aceptaron participar firmaron un consentimiento informado, en el que se especificó el carácter voluntario de la participación en este estudio, la posibilidad de abandonar la investigación en cualquier momento sin ningún 
impedimento por parte de los investigadores, así como la utilización de sus datos de manera anónima y confidencial. El primer día de sesión, la musicoterapeuta enfatizó la importancia de que todas las participantes se comprometieran a respetar y preservar la confidencialidad de los aspectos abordados durante la sesión ya fuera con respecto a sí mismas o de las otras compañeras del grupo. Un aspecto importante, es el hecho de que una vez acabado el taller, las participantes hayan podido integrar alguno de los diferentes recursos de apoyo y acompañamiento que la música puede ofrecerles. El objetivo es que las pacientes puedan continuar utilizando y apoyándose en la música y experiencias musicales en su día a día como un recurso personal más de apoyo, y facilitar oportunidades para desarrollar un mayor grado de autonomía y autocontrol emocional. Además, se pretendía también generar nuevas redes sociales entre pacientes que están pasando por la misma enfermedad.

\section{Análisis estadístico}

Para llevar a cabo el análisis estadístico se empleó el paquete estadístico IBM SPSS Statistics versión 21. Se consideró estadísticamente significativa aquella diferencia con un nivel de significación de $\mathrm{p}<0,05$.)

\section{RESULTADOS}

Destacamos, en primer lugar, el elevado porcentaje de asistencia de las participantes a las sesiones: Cinco de las 8 pacientes han asistido al $100 \%$ de las sesiones (8 sesiones), 2 pacientes han asistido al $87,50 \%$ de las sesiones (7 sesiones) y 1 de ellas ha asistido al $75 \%$ de las sesiones (6 sesiones). Mostramos a continuación los resultados obtenidos en relación a cada una de las variables estudiadas.

\section{a) Resultados pre/post tratamiento}

- Estado de Ánimo

Se analizaron las diferencias halladas entre las medias de las puntuaciones pre/ post tratamiento en cuanto a los niveles de Ansiedad y Depresión de la escala HADS y no muestran diferencias significativas. Como puede observarse en la tabla 2, las puntuaciones de Depresión del total de la muestra, no entran dentro del rango clínico depresión clínica (0-7) ni antes ni después del tratamiento. Con respecto a las puntuaciones de Ansiedad obtenidas antes del tratamiento, éstas se ubican dentro del rango calificado como caso clínico dudoso, y disminuyen después del tratamiento, pasando a considerarse caso normal, sin problema clínico.

Por otro lado, las medias de las puntuaciones de la Escala POMS-A muestran un cambio estadísticamente significativo en el Índice de Alteración Emocional antes y después del tratamiento. Todas las subescalas muestran una disminución de las puntuaciones al finalizar el tratamiento; no obstante, la subescala Tensión es la única que disminuye significativamente $(p<0,05)$. Por otro lado, en el caso de la subescala Vigor, esta disminución representa un "empeoramiento" del síntoma dado que, a diferencia de las otras cuatro, se trata de una variable positiva. La tabla 3 recoge las medias de las puntuaciones de este Índice.

\section{- Calidad de Vida:}

Para el análisis de la Calidad de Vida en este estudio se han utilizado las 6 dimensiones principales del cuestionario de Calidad de Vida de la EORTC-QLQ-30: la Escala de Calidad de Vida Global y las 5 Escalas de Funcionamiento. No se han administrado las 3 Escalas de Síntomas. Los resultados no muestran cambios significativos (ver la tabla 4). 
Tabla 2. Media de las puntuaciones en Ansiedad y Depresión (HADS) Pre /Post-Tratamiento

\begin{tabular}{|c|c|c|c|c|c|}
\hline \multirow{2}{*}{ HADS } & \multicolumn{2}{|c|}{$\begin{array}{c}\text { Pre } \\
\text { Tratamiento }\end{array}$} & \multicolumn{2}{c|}{$\begin{array}{c}\text { Post } \\
\text { Tratamiento }\end{array}$} & \\
\hline Subescala & Media & DT & Media & DT & p \\
\hline Ansiedad & 8,75 & 6,319 & 7,63 & 4,069 & 0,500 \\
\hline Depresión & 5,75 & 4,464 & 5,63 & 3,292 & 0,526 \\
\hline
\end{tabular}

HADS: Escala de Ansiedad Hospitalaria.

Tabla 3. Media de las puntuaciones en Índice Alteración Emocional y Subescalas (POMS-A) Pre / Post-Tratamiento

\begin{tabular}{|c|c|c|c|c|c|}
\hline \multirow{2}{*}{$\begin{array}{c}\text { POMS-A } \\
\text { Subescala }\end{array}$} & \multicolumn{2}{|c|}{ Pre-Tratamiento } & \multicolumn{2}{|c|}{ Post-Tratamiento } & \multirow[b]{2}{*}{ p } \\
\hline & Media & DT & Media & DT & \\
\hline Índice Alteración Emocional & 24,38 & 13,469 & 17,63 & 6,948 &, $028^{*}$ \\
\hline Tensión & 5,13 & 3,137 & 3,00 & 2,268 &, $041^{*}$ \\
\hline Fatiga & 4,00 & 3,381 & 1,50 & 1,604 &, 058 \\
\hline Depresión & 5,25 & 4,062 & 4,25 & 2,964 &, 129 \\
\hline Cólera & 4,00 & 2,726 & 2,25 & 1,488 & ,088 \\
\hline Vigor & 6,00 & 2,619 & 5,38 & 2,973 & ,892 \\
\hline
\end{tabular}

POMS-A: Escala Profile of Humor States.

* Diferencia significativa $(\mathrm{p}<0,05)$

Tabla 4. Media de las Puntuaciones en Calidad de Vida (EORTC-QLQ-30) Pre / Post-Tratamiento

\begin{tabular}{|l|l|l|l|l|l|}
\hline \multicolumn{1}{|c|}{ EORTC } & \multicolumn{2}{c|}{ Pre-Tratamiento } & \multicolumn{1}{c|}{ Post-Tratamiento } & \multicolumn{1}{c}{ DT } & \multicolumn{1}{c|}{ p } \\
\hline \multicolumn{1}{|c|}{ Subescala } & \multicolumn{1}{|c|}{ Media } & \multicolumn{1}{c|}{ DT } & \multicolumn{1}{c|}{ Media } & \multicolumn{1}{c|}{ DT } \\
\hline Calidad de Vida Global & 80,13 & 22,95 & 80,00 & 19,27 & 0,888 \\
\hline Funcionamiento Físico & 77,00 & 16,239 & 71,38 & 22,627 & 0,246 \\
\hline Funcionamiento de Rol & 69,00 & 22,860 & 71,13 & 30,624 & 1,000 \\
\hline Funcionamiento Emocional & 52,25 & 27,139 & 65,75 & 28,724 & 0,027 \\
\hline Funcionamiento Cognitivo & 64,75 & 24,575 & 67,00 & 18,174 & 0,581 \\
\hline Funcionamiento Social & 73,13 & 23,607 & 75,25 & 19,962 & 0,581 \\
\hline
\end{tabular}

EORTC: Cuestionario de Calidad de Vida de la EORTC-QLQ-30. 


\section{b) Resultados pre/post sesión}

Para evaluar los cambios en el estado de ánimo pre-post sesión, se llevó a cabo la prueba de Wilcoxon de los rangos con signo de muestras relacionadas. El análisis del efecto global de la intervención, que compara para cada ítem la media global de todas las puntuaciones medias de EVN a nivel pre-sesión con la media global de todas las puntuaciones medias de EVN a nivel post-sesión, muestra un efecto significativo de la intervención en cuanto a disminución de los niveles de Ansiedad Global, Tristeza Global y Malestar Físico Global antes y después de la sesión. Si bien el grado de asistencia ha sido muy elevado, en este análisis han sido incluidos únicamente aquellos 5 sujetos que han asistido a la totalidad de las sesiones (8) y no se han incluido a 3 participantes que han faltado a 1 o 2 sesiones únicamente). La tabla 5 recoge estos resultados.

Por otro lado, si analizamos el efecto de la intervención en cada sesión, los resultados muestran una disminución significativa de los niveles de Ansiedad, Tristeza y Malestar Físico para la mayoría de las sesiones. Los niveles de Ansiedad disminuyeron en todas las sesiones, los de Malestar Físico en todas excepto en la sesión $n^{\circ} 5$, y los de Tristeza se disminuyeron únicamente en la mitad de las sesiones (sesión $n^{\circ} 4,5,7$ y 8 ). La tabla 6 muestra los descriptivos así como la prueba de Wilcoxon y su grado de significancia para cada uno de estos 3 ítems y especifica el número de participantes asistentes a cada sesión.

\section{c) Resultados Cuestionario de Valoración}

El Cuestionario de Valoración del Programa de Musicoterapia, elaborado ad hoc, ha permitido explorar varios aspectos. Con respecto a la percepción de la mejora del estado anímico para cada una de las sesiones se han obtenido los siguientes resultados: Seis de las participantes expresan que este programa les ha ayudado a mejorar "Mucho", mientras que 2 de ellas consideran que han mejorado "Bastante". En cuanto a la mejora del estado anímico en su día a día (y, por tanto, en situaciones y momentos fuera de la sesión), ésta se produce tanto con respecto a la ansiedad como a la tristeza: la mayoría de participantes $(n=6)$ sostienen que les ha ayudado "Bastante" a disminuir sus estados de tristeza, mientras que 1 de ellas considera que "Mucho" y otra que "Un poco". La ayuda para mejorar los estados de ansiedad en el día a día ha sido percibida como "Bastante" $(n=4)$, "Mucho" $(n=3)$ y "Un poco" $(n=1)$.

Además, a través de este cuestionario ha sido posible recoger otros aspectos del programa de musicoterapia que pueden haber contribuido a esta mejora del estado de ánimo. Uno de ellos tiene que ver con la expresión de emociones y sentimientos: Las puntuaciones revelan que la mayoría de personas $(n=7)$ consideran que este programa les ha ayudado "Mucho" a expresar emociones y sentimientos, mientras que para una de ellas esta ayuda ha contribuido en sólo "Un poco". Hablar de la enfermedad ha sido un aspecto abordado en las sesiones que puede haber contribuido también a esta expresión y ventilación emocional. Las participantes consideran que esta experiencia les ha dado la oportunidad de hablar "Bastante" $(n=5)$, "Mucho" $(n=2)$ y "Un poco" $(n=1)$. Por otro lado, según las respuestas de las participantes, el programa les ha permitido además, distraerse y evadirse de las cosas que les preocupan "Mucho" $(n=5)$ y "Bastante" ( $n=3)$ así como relajarse y soltar tensiones "Mucho" $(\mathrm{n}=6)$ y "Bastante" $(n=2)$. Finalmente destacar que el formato grupal del programa ha contribuido a la socialización, aspecto que ha quedado también recogido en el Cuestionario de 


\section{Tabla 5. Descriptivos y Prueba de Wilcoxon para EVN Global de Ansiedad, Tristeza y Malestar Físico Pre/Post Sesión ( $\mathbf{N = 5}$ )}

\begin{tabular}{|c|c|c|c|c|c|c|c|c|c|}
\hline \multirow{2}{*}{$\begin{array}{c}\text { EVN } \\
\text { Global } \\
\text { Sesiones }\end{array}$} & \multicolumn{3}{|c|}{ Ansiedad } & \multicolumn{3}{c|}{ Tristeza } & \multicolumn{3}{c|}{ Malestar Físico } \\
\cline { 2 - 9 } & $\begin{array}{c}\text { Pre-M } \\
\text { (DT) }\end{array}$ & $\begin{array}{c}\text { Post-M } \\
\text { (DT) }\end{array}$ & $\mathbf{P}$ & $\begin{array}{c}\text { Pre-M } \\
\text { (DT) }\end{array}$ & $\begin{array}{c}\text { Post-M } \\
\text { (DT) }\end{array}$ & $\mathbf{p}$ & $\begin{array}{c}\text { Pre-M } \\
\text { (DT) }\end{array}$ & $\begin{array}{c}\text { Post-M } \\
\text { (DT) }\end{array}$ & $\mathbf{p}$ \\
\hline $\begin{array}{c}\text { Sesión } \\
\mathbf{1} \text { a 8 }\end{array}$ & $\begin{array}{c}2,72 \\
(1,587)\end{array}$ & $\begin{array}{c}1,32 \\
(1,418)\end{array}$ &, $042 *$ & $\begin{array}{c}2,40 \\
(1,534)\end{array}$ & $\begin{array}{c}1,17 \\
(1,133)\end{array}$ &, $042 *$ & $\begin{array}{c}2,87 \\
(1,697)\end{array}$ & $\begin{array}{c}1,70 \\
(1,480)\end{array}$ &, $039^{*}$ \\
\hline
\end{tabular}

* Diferencia significativa $(\mathrm{p}<0,05)$.

Tabla 6. Descriptivos y Prueba de Wilcoxon para EVN de Ansiedad, Tristeza y Malestar Físico Pre/Post Sesión

\begin{tabular}{|c|c|c|c|c|c|c|c|c|c|}
\hline \multirow{2}{*}{$\begin{array}{c}\text { EVN } \\
\text { por } \\
\text { sesiones }\end{array}$} & \multicolumn{3}{|c|}{ Ansiedad } & \multicolumn{3}{c|}{ Tristeza } & \multicolumn{3}{c|}{ Malestar Físico } \\
\cline { 2 - 9 } & Pre-M & $\begin{array}{c}\text { Post-M } \\
(\mathbf{D T})\end{array}$ & $\mathbf{p}$ & $\begin{array}{c}\text { Pre-M } \\
(\mathbf{D T})\end{array}$ & $\begin{array}{c}\text { Post-M } \\
(\mathbf{D T})\end{array}$ & $\mathbf{p}$ & $\begin{array}{c}\text { Pre-M } \\
\text { (DT) }\end{array}$ & $\begin{array}{c}\text { Post-M } \\
(\mathrm{DT})\end{array}$ & $\mathbf{p}$ \\
\hline $\begin{array}{c}\text { Sesión 1 } \\
(\mathrm{N}=6)\end{array}$ & $\begin{array}{c}3,00 \\
(2,000)\end{array}$ & $\begin{array}{c}1,67 \\
(1,633)\end{array}$ &, $038^{*}$ & $\begin{array}{c}3,17 \\
(1,835)\end{array}$ & $\begin{array}{c}1,83 \\
(1,941)\end{array}$ &, 071 & $\begin{array}{c}4,00 \\
(1,414)\end{array}$ & $\begin{array}{c}2,00 \\
(1,265)\end{array}$ &, $042^{*}$ \\
\hline $\begin{array}{c}\text { Sesión 2 } \\
(\mathrm{N}=8)\end{array}$ & $\begin{array}{c}3,38 \\
(2,973)\end{array}$ & $\begin{array}{c}1,25 \\
(1,832)\end{array}$ &, $017^{*}$ & $\begin{array}{c}2,50 \\
(3,024)\end{array}$ & $\begin{array}{c}0,75 \\
(1,165)\end{array}$ &, 066 & $\begin{array}{c}3,75 \\
(3,370)\end{array}$ & $\begin{array}{c}1,75 \\
(1,982)\end{array}$ &, $041^{*}$ \\
\hline $\begin{array}{c}\text { Sesión 3 } \\
(\mathrm{N}=7)\end{array}$ & $\begin{array}{c}1,71 \\
(1,604)\end{array}$ & $\begin{array}{c}0,86 \\
(1,215)\end{array}$ &, $034^{*}$ & $\begin{array}{c}1,86 \\
(2,035)\end{array}$ & $\begin{array}{c}1,00 \\
(1,414)\end{array}$ &, 063 & $\begin{array}{c}2,14 \\
(1,773)\end{array}$ & $\begin{array}{c}1,14 \\
(1,676)\end{array}$ &, $038^{*}$ \\
\hline $\begin{array}{c}\text { Sesión 4 } \\
(\mathrm{N}=8)\end{array}$ & $\begin{array}{c}2,13 \\
(1,458)\end{array}$ & $\begin{array}{c}0,63 \\
(1,061)\end{array}$ &, $026^{*}$ & $\begin{array}{c}1,88 \\
(1,642)\end{array}$ & $\begin{array}{c}0,63 \\
(1,061)\end{array}$ &, $041^{*}$ & $\begin{array}{c}3,00 \\
(3,071)\end{array}$ & $\begin{array}{c}1,88 \\
(2,100)\end{array}$ &, $038^{*}$ \\
\hline $\begin{array}{c}\text { Sesión 5 } \\
(\mathrm{N}=8)\end{array}$ & $\begin{array}{c}2,13 \\
(1,356)\end{array}$ & $\begin{array}{c}0,88 \\
(1,727)\end{array}$ &, $040^{*}$ & $\begin{array}{c}1,75 \\
(1,488)\end{array}$ & $\begin{array}{c}0,63 \\
(1,408)\end{array}$ &, $038^{*}$ & $\begin{array}{c}2,50 \\
(2,390)\end{array}$ & $\begin{array}{c}1,50 \\
(1,773)\end{array}$ &, 066 \\
\hline $\begin{array}{c}\text { Sesión 6 } \\
(\mathrm{N}=8)\end{array}$ & $\begin{array}{c}2,75 \\
(2,493)\end{array}$ & $\begin{array}{c}1,13 \\
(1,553)\end{array}$ &, $027^{*}$ & $\begin{array}{c}1,75 \\
(2,315)\end{array}$ & $\begin{array}{c}0,63 \\
(1,188)\end{array}$ &, 066 & $\begin{array}{c}3,25 \\
(2,816)\end{array}$ & $\begin{array}{c}1,88 \\
(2,232)\end{array}$ &, $016^{*}$ \\
\hline $\begin{array}{c}\text { Sesión 7 } \\
(\mathrm{N}=8)\end{array}$ & $\begin{array}{c}1,50 \\
(1,604)\end{array}$ & $\begin{array}{c}0,38 \\
(, 518)\end{array}$ &, $041^{*}$ & $\begin{array}{c}1,63 \\
(1,188)\end{array}$ & $\begin{array}{c}0,50 \\
(0,535)\end{array}$ &, $034^{*}$ & $\begin{array}{c}2,13 \\
(1,808)\end{array}$ & $\begin{array}{c}1,00 \\
(1,195)\end{array}$ &, $041^{*}$ \\
\hline $\begin{array}{c}\text { Sesión 8 } \\
(\mathrm{N}=8)\end{array}$ & $\begin{array}{c}1,75 \\
(1,035)\end{array}$ & $\begin{array}{c}0,75 \\
(1,035)\end{array}$ &, $020^{*}$ & $\begin{array}{c}1,50 \\
(1,414)\end{array}$ & $\begin{array}{c}0,63 \\
(0,916)\end{array}$ &, $038^{*}$ & $\begin{array}{c}2,00 \\
(1,309)\end{array}$ & $\begin{array}{c}1,00 \\
(1,069)\end{array}$ &, $023^{*}$ \\
\hline
\end{tabular}

$*$ Diferencia significativa $(p<0,05)$.

Valoración, con unas puntuaciones que reflejan un alto grado de ayuda de este programa con respecto a relacionarse y hablar con las compañeras del grupo: Siete participantes la puntúan como "Mucho" y una como "Bastante".

Este cuestionario muestra una total unanimidad en cuanto a recomendar esta experiencia a otras participantes ("Mucho" $\left.^{\prime \prime} \mathrm{n}=8\right)$ y un alto grado de utilidad del mismo: ("Mucho", $\mathrm{n}=6$ y "Bastante", $\mathrm{n}=2$ ).

\section{DISCUSIÓN}

Cuidar las necesidades de las mujeres supervivientes de un cáncer de mama es 
un reto importante en la actualidad. El desarrollo de diferentes modalidades terapéuticas a nivel psicosocial permitirán contribuir a una mejora de su malestar emocional y de su calidad de vida, entre otros aspectos. El objetivo de este estudio se ha centrado en explorar la eficacia de un tratamiento con musicoterapia para mejorar el estado anímico y calidad de vida de un grupo de mujeres que se encuentran en fase de supervivencia de un cáncer de mama.

Tras el análisis de datos de nuestro estudio, y con respecto a la variable estado de ánimo a nivel de post-tratamiento, vemos como el programa de musicoterapia ha tenido una influencia interesante. Los resultados del HADS reflejan una disminución en las puntuaciones de sintomatología ansioso-depresiva, aunque sin Ilegar a ser significativas. Tal y como se ha comentado ya en el apartado de Resultados, una posible explicación es que la media de las puntuaciones a nivel pre-tratamiento de la variable Depresión se sitúan en un rango de normalidad, de no patología clínica, y disminuye un poco tras el tratamiento. Con respecto a la variable Ansiedad, la media de las puntuaciones pre-tratamiento se sitúan ligeramente dentro del rango de caso clínico dudoso. Si analizamos los datos a nivel individual, observamos que hay 4 sujetos que presentan problemas clínicos a nivel pre-tratamiento en cuanto a Ansiedad, y que a nivel post-tratamiento disminuyen a un nivel de normalidad en 1 caso, en 2 casos se sitúa en el rango de caso clínico dudoso, y en otro de ellos, si bien se reduce también, se mantiene dentro del rango de caso con problema clínico. Con respecto al análisis individual de la variable Depresión, se detectan únicamente 2 casos clínicos, que disminuyen en el posttratamiento, uno de ellos se sitúa como caso dudoso y el otro se mantiene como caso con problema clínico. La muestra de este estudio presenta unos niveles de dis- trés emocional (ansiedad y depresión) más bien bajos; los resultados del HADS son consistentes con algunas de las evidencias que constatan que aquellos pacientes con niveles altos de ansiedad o distrés responden más favorablemente a la musicoterapia que aquellos con niveles más bajos ${ }^{(51)}$, y en nuestro caso, no parece haber pues tanta presencia de sintomatología a la que tratar con una intervención.

El POMS-A refleja una disminución significativa en el Índice de Alteración Emocional. Cabe decir que de los 8 sujetos, 5 se sitúan a nivel pre-tratamiento en puntuaciones bajas, mostrando poco malestar emocional, siendo 3 las que se sitúan en puntuaciones medias de este índice. Al final del tratamiento (post-) se puede observar que todos los sujetos muestran unas puntuaciones bajas y solo uno de ellos se mantiene en rangos medios, pese a haber reducido igualmente su malestar emocional. Este resultado es consistente con estudios previos de musicoterapia en cáncer, en el que se observa una mejora significativa en el estado de ánimo como resultado de participar en sesiones de musicoterapia $^{(73,83)}$. El análisis de las subescalas de este instrumento nos muestra como la subescala Tensión disminuye significativamente, mientras que las subescalas Fatiga, Depresión, Cólera y Vigor, si bien también disminuyen tras finalizar el tratamiento, no lo hacen de manera significativa. No obstante, y con respecto a la subescala Vigor, cabe señalar que ésta es una variable positiva dentro del POMS-A, y que su disminución no representaría precisamente una mejora en el estado anímico de estas participantes y por tanto no lleva a un cambio en la dirección deseada. Por otra parte, y en el caso de esta escala, podemos decir que la tendencia de las puntuaciones obtenidas en cada subescala va en la línea de los resultados de la revisión Cochrane de Bradt, Dileo, Grocke \& Magill(84) en el contexto oncológico que sugieren que la 
variable depresión o fatiga no responde de manera tan clara ante las intervenciones con musicoterapia, como sí lo hace la ansiedad (Tensión) de los pacientes con cáncer. Por otro lado, y tal y como señaló Waldon $^{(83)}$ en su estudio hemos de tener en cuenta que los estados de ánimo son fluctuantes por naturaleza, y el POMS-A es un instrumento sensitivo a las manifestaciones anímicas concurrentes.

En relación a la variable Calidad de Vida, los resultados no muestran cambios significativos para la Escala Calidad de Vida Global de la EORTC-QLQ-30. De las 5 Subescalas de Funcionamiento, únicamente se ha obtenido un cambio estadísticamente significativo en la subescala de Funcionamiento Emocional. La dimensión emocional es un aspecto en el que se ha incidido especialmente en este programa de musicoterapia, y podría haber conducido a esta mejora funcional. No obstante, hemos de ser cautos al respecto, dado que somos conscientes de que a lo largo de estos 2 meses de tratamiento y durante cada semana, han podido confluir otras variables no controladas que promuevan esta mejora. Se desestimó aplicar la Escala de Síntomas Físicos por el hecho de que este estudio va directamente dirigido a trabajar el estado anímico y porque los estudios clínicos en cáncer muestran un mayor impacto en aquellas variables que hacen referencia a respuestas emocionales que en las de síntomas físicos. Otra razón importante para esta decisión ha sido, además, la de reducir el tiempo de administración de la batería de tests pre/post tratamiento a las participantes.

Los resultados que la revisión Cochrane recoge para la variable calidad de vida muestran una mejora en ésta, si bien sin diferencias significativas ${ }^{(84)}$. Esto puede explicarse por el hecho de que frecuentemente, la calidad de vida es una variable que viene asociada a otras, ya sean relacionadas con aspectos físicos (dolor, relajación, tensión, etc.) o psicológicos (ansiedad, tristeza,... etc.). El estrés y la ansiedad pueden disminuir y el estado de ánimo y la calidad de vida mejorar, como resultado de intervenciones con musicoterapia activa o receptiva. Se puede observar, por tanto, que las intervenciones musicoterapéuticas tienen una influencia en los procesos psicológicos que a su vez afectan y son afectados por procesos físicos y sociales ${ }^{(85)}$. En esta línea consideramos adecuado comentar que el Cuestionario de Valoración del programa ha recogido algunas de descripciones de la experiencia vivida en este programa que creemos que podrían ir relacionadas con lo que sería una mejora en su calidad de vida. Recogemos seguidamente algunas de estas vivencias: "Esta experiencia me ha aportado tranquilidad, confianza y mucha paz...", "Un espacio para compartir y para cuidarse.", "Gratificante, divertida y, sobre todo, saludable., "Ha sido una experiencia positiva, muy positiva...", "Enriquecedora.", "Muy buena.", "A mi me ha hecho mucho bien y lo encontraré a faltar. Para mí todo ha sido perfecto.", "Este taller ha sido estupendo, desde la terapeuta hasta mis compañeras."

Por otro lado, este estudio ha tratado de ver también el efecto de este tratamiento de musicoterapia en el estado de ánimo de las participantes a nivel de post-sesión a través de la EVN, de forma que si observamos los resultados de las puntuaciones medias de los ítems de Ansiedad, Tristeza y Malestar Físico de manera global (sesión 1 a 8 de manera compactada), estos muestran una significación para los 3 ítems, lo que nos lleva a afirmar que esta intervención ha resultado efectiva para disminuir la percepción del malestar emocional y físico de las participantes. Esta disminución se puede observar también en el análisis por separado de cada sesión: después de cada sesión se ha producido siempre una disminución en las puntuaciones de las EVN. Destaca el hecho de que todas las sesiones 
han resultado ser efectivas para reducir los niveles de malestar emocional en cuanto a Ansiedad y Tristeza. Las técnicas de expresión emocional y comunicación, como son el análisis lírico de canciones, la composición musical, así como las audiciones musicales y cantar canciones, han permitido expresar emociones y sentimientos a nivel verbal y no verbal, y canalizarlos a través de experiencias musicales. Esta capacidad de la musicoterapia para disminuir el malestar emocional y mejorar el estado anímico ha mostrado una disminución significativa de la Ansiedad en las 8 sesiones que configuran el programa $y$, si bien la disminución de los niveles de Tristeza se ha dado en todas ellas, ésta solo se ha producido significativamente en la mitad (sesión 4, 5, 7 y 8). Una posible explicación al respecto podría ser el hecho de que los niveles de tristeza iniciales, con los que llegan las participantes a la sesión, no son excesivamente altos, y se sitúan algo más por debajo que los niveles iniciales de malestar físico o incluso ansiedad. Paralelamente, y como hemos comentado anteriormente, existe evidencia de que la variable Depresión (y por extensión, la Tristeza) no suele ser tan sensible a este tipo de intervenciones. La persistencia de las secuelas físicas en pacientes supervivientes de cáncer de mama es un hecho. Al tratarse de pacientes que han pasado por una experiencia de tratamientos oncológicos, se decidió incluir el ítem Malestar Físico en este estudio y considerarla como una variable que, si bien no incide de manera directa con el estado de ánimo, sí que puede modularlo e influenciarlo ${ }^{(85-88)}$. Se ha reducido este tipo de malestar en todas las sesiones, y esta disminución ha resultado ser significativa en 7 de las 8 sesiones que configuran el programa.

Así pues, y en lo que respecta a la batería de tests estandarizados que han sido administrados en este estudio así como a la EVN, podemos ver cómo, a excepción del HADS, todos ellos presentan resultados similares para las variables Ansiedad y Tensión así como para la variable Depresión y Tristeza. Ello nos permite hablar de una mejora emocional y, por tanto, una mejora del estado anímico de estas participantes del programa de musicoterapia.

Es interesante destacar el alto grado de utilidad de este programa que ha percibido cada asistente y que ha reflejado en su Cuestionario de Valoración. Creemos que estas EVN que las participantes se auto-administran antes y después de cada sesión, les ofrece una oportunidad de conectar consigo mismas, de observar el propio estado emocional y físico de ese momento, y de tomar consciencia de los cambios que se puedan producir al finalizar la sesión. Ello muy probablemente les haya podido ayudar a darse cuenta de que implicarse activamente en algo puede mejorar su insight, aumentar su sensación de control, y tomar decisiones orientadas hacia el cuidado de sí mismas. Ello nos lleva a confirmar, una vez más, la importancia de incorporar programas y tratamientos que tengan en cuenta las necesidades de este colectivo, y que permitan mostrarles nuevos recursos que puedan resultar útiles en su camino de recuperación y readaptación a la "vida normal" con los retos que les demanda su día a día.

Otro aspecto observado a lo largo del programa ha sido el alto grado de asistencia y participación de los sujetos. La adherencia al tratamiento de musicoterapia y, por tanto, la asistencia continuada a las sesiones por parte de este grupo de pacientes en fase de supervivencia contrasta con el alto grado de ausencias y/o abandono que se suelen dar en aquellos estudios que cuentan con pacientes oncológicos que están en tratamiento activo y que no pueden asistir de manera regular o incluso llegan a abandonar, ya sea por la progresión de la enfermedad, empeoramiento de síntomas y limitaciones, hospitalización, 
solapamiento con otras pruebas o visitas médicas, etc. ${ }^{(46,49,89,90)}$. Con respecto a la participación, si bien en los momentos iniciales de la intervención, los participantes de un grupo de musicoterapia pueden mostrar cierta reticencia e inseguridad ante propuestas musicoterapéuticas más interactivas y de mayor grado de implicación y participación, estas experiencias pueden acabar resultando agradables, terapéuticas y beneficiosas. Así ha sucedido en este grupo. Para ello es importante la presencia de un musicoterapeuta profesional, especializado no solo para adaptar la música y experiencias musicales al perfil de pacientes a los que se dirige, sino también para ayudar a canalizar, expresar y reconducir adecuadamente aquellas respuestas de cada participante y del grupo. Tal y como se recomienda en la revisión Cochrane, este programa ha utilizado no solo técnicas pasivo-receptivas sino también técnicas activas a fin de poder trabajar adecuadamente las necesidades del paciente oncológico y los correspondientes objetivos terapéuticos ${ }^{(84)}$. Es de suma importancia ofrecer una variedad de técnicas y actividades, dado que las respuestas individuales a la música son diferentes según cada persona, y porque así se ofrecen nuevas y diferentes vías de exploración y utilización de la música de manera terapéutica en el día a día. En este grupo, las técnicas que han tenido mejor acogida, y de manera unánime, han sido la técnica de análisis lírico y tocar instrumentos de percusión. Algo por debajo, aunque también bien recibidas también han sido las técnicas de cantar canciones con las compañeras y practicar ejercicios de relajación acompañados de música. Analizar las letras de canciones con un mensaje significativo ha permitido explorar ciertas cuestiones y reflexionar sobre la propia manera de pensar, sentir y actuar, y observar otras estrategias y maneras de afrontamiento de las compañeras que pueden ser diferen- tes. Las distintas actividades de relajación aplicadas han generado un espacio más personal e íntimo durante las sesiones, en el que se favorece más el contacto con uno mismo, pese a estar en contexto grupal. En cada sesión se han trabajado estrategias de relajación acompañada con música a fin de reducir la posible tensión muscular o dolor que presentaran las participantes. Paralelamente, otra manera de promover estados de distensión ha sido precisamente con técnicas de carácter activo, tales como tocar instrumentos musicales de pequeña percusión que han requerido la focalización en la tarea musical así como de sincronización de movimientos con la música, técnicas que han ofrecido una estrategia de canalización y liberación de este malestar físico, así como una posible distracción del mismo. Además, las actividades de tocar instrumentos o cantar en grupo permiten crear un espacio de mayor complicidad y sincronización entre las participantes, de comunicación a nivel no verbal, hecho que ayuda también a la cohesión del grupo ${ }^{(14,16,68,83,90)}$.

Somos conscientes de que una de las limitaciones de este estudio es el tamaño pequeño de la muestra. Sabemos que esto sucede frecuentemente en estudios realizados en el campo de la musicoterapia oncológica. Queremos matizar, no obstante, que se trata de un programa enmarcado como proyecto piloto en ese hospital, y que permite obtener unos resultados preliminares sobre los que tomar decisiones posteriores a nivel de adaptaciones, cambios, e incluso seguimiento y continuidad del mismo. Además del tamaño pequeño de la muestra, este estudio no ha contado con una muestra aleatorizada, carece de grupo control. Presenta, además, también problemas de heterogeneidad de la muestra, que pueden ser atribuidos al proceso de derivación y a unos criterios de inclusión demasiado amplios. En cuanto a las variables clínicas, el periodo de tiem- 
po transcurrido desde el fin de tratamiento activo es demasiado amplio, y debería acotarse más, dado que el estado y necesidades de una paciente que se encuentra a 3 meses de haber finalizado el tratamiento activo puede ser muy distinto del estado y necesidades de otra que hace 18 o 24 meses que han acabado. Unificar la muestra con respecto a la variable tratamiento con terapia hormonal ayudaría a controlar mejor el efecto que este tratamiento suele tener a nivel físico y psicológico, y a abordar o no estos aspectos en la sesión. De cara a futuros estudios, también conviene reflexionar y considerar la posibilidad de establecer como criterio de inclusión a aqueIlos sujetos que presentan un mayor grado de malestar emocional en cuanto a nivel de ansiedad y depresión, por las razones expuestas anteriormente. Con respecto a ajustes en aspectos relacionados con las variables sociodemográficas, creemos que se debería homogeneizar todavía más la muestra especialmente en cuanto a edad y situación laboral. En nuestro estudio hay mucha diversidad en cuanto al rango de edad y situación laboral, entre otros, con las consecuentes realidades que ello comporta (tener hijos o no, tener pareja o ser viuda, estar trabajando o de baja vs. estar jubilada...). Un grupo más homogéneo en cuanto a estas variables permitiría abordar determinadas cuestiones y preocupaciones en la sesión de una manera más directa y adaptada a las necesidades de cada participante, como serían cambios en la dinámica familiar, alteraciones sexuales, dificultades para reincorporarse a la vida laboral, cambios en estilo de vida, dependencia, comunicación y relaciones sociales, etc.

Pese a estas limitaciones, nos parece importante atribuir buena parte de ellas al hecho de que se trata de un estudio en el marco de una experiencia inicial y piloto, que ha permitido aplicar esta modalidad de tratamiento, explorar los beneficios del mismo así como detectar aquellos puntos a mejorar y a ajustar. Como punto fuerte e interesante de esta experiencia, podemos decir que ha incluido la figura de un musicoterapeuta profesional, hecho que ha permitido diseñar y adaptar las intervenciones al perfil y necesidades de las participantes, ha contemplado las preferencias musicales de cada una de ellas, y ha incorporado técnicas de musicoterapia de tipo activo y receptivo, en un formato de grupo que ha ayudado a abordar aspectos sociales y relacionales de las participantes. Además, si revisamos las experiencias realizadas con pacientes oncológicos, muchas de ellas se basan en intervenciones de una sesión o dos; pocas de ellas ofrecen una continuidad de 8 sesiones semanales de 90 minutos de duración. El efecto de la intervención de musicoterapia ha sido cuidado mediante el criterio de únicamente incluir en el estudio a aquellos sujetos que hayan asistido al menos al 50\% de las sesiones; este es un aspecto que no queda clara ni suficientemente recogido en los estudios de musicoterapia realizados en este campo. Un aspecto interesante también es el haber podido implementar el tratamiento en el propio hospital de referencia de las pacientes y ofrecer a las participantes una experiencia no invasiva, agradable y gratificante, en un entorno que hasta la fecha ellas consideraban duro, hostil e invasivo. Finalmente destacar el hecho de haber centrado en esta fase de supervivencia. Por dos razones importantes: la primera el hecho de que el carácter crónico del cáncer está Ilevando a nuestra sociedad a nuevas necesidades y retos de atención al paciente superviviente de cáncer. Esto significa la posibilidad de incluir modalidades de intervención desde las terapias complementarias. La segunda razón, es la de poder contribuir con este estudio con nueva información al bagaje teórico y clínico del campo de la psicooncología y de la musicoterapia, dos disciplinas que actualmente disponen de pocos estudios realizados con personas en fase de supervivencia. 


\section{CONCLUSIONES}

El incremento actual de la supervivencia de las pacientes con cáncer de mama está derivando en una emergente necesidad de desarrollar nuevas estrategias terapéuticas e incorporar aquellas modalidades de intervención que permitan dar respuesta de una manera global a las diferentes dificultades que conlleva ser una mujer superviviente de un cáncer de mama. Los resultados de este estudio sugieren que la musicoterapia puede producir un cambio positivo en el estado de ánimo de este perfil de participantes, no sólo en cuanto a disminuir su malestar emocional durante la sesión, sino también a lo largo del tratamiento. La variable calidad de vida, si bien no ha reflejado un gran cambio, sí que muestra un cambio significativo en su dimensión emocional. Es necesario seguir investigando en el campo de la musicoterapia y de la psicooncología a fin de poder seguir documentando nuevos hallazgos y contribuyendo a mejorar el estado de ánimo y calidad de vida de mujeres con cáncer en esta fase de supervivencia.

\section{Agradecimientos}

Deseamos expresar nuestro agradecimiento a la Fundació Oncolliga - Lliga Catalana d'Ajuda Oncològica por el apoyo prestado. Agradecemos a la Unidad de Patología Mamaria del Hospital de Mar, que ha contribuido al desarrollo de este proyecto piloto. Nuestro agradecimiento muy especial también a la Dra. Montserrat Freixa. Por último, dar las gracias a las mujeres que han participado en este estudio, por su confianza, implicación y entusiasmo con la música y la vida.

\section{REFERENCIAS BIBLIOGRÁFICAS}

1. Sociedad Española de Oncología Médica. Las Cifras del Cáncer en España
2014. 2014; [Internet]. [Acceso el 10 de marzo de 2015] Disponible en: http:// www.seom.org/en/prensa/el-cancer-enespanyacom/104582-el-cancer-en-espana-2014

2. Die Trill M, Vecino S. El Superviviente de cáncer. Psico-oncologia. Madrid: ADES Ediciones; 2003. p. 125-41.

3. Andrés Solana C. Aspectos psicológicos en el paciente superviviente. Oncología 2005;28:51-7.

4. Andrykowski MA, Lykins E, Floyd A. Psychological health in cancer survivors. Semin Oncol Nurs 2008;24:193-201. Doi:10.1016/j.soncn.2008.05.007

5. Grupo Español de Pacientes con Cáncer GEPAC. Informe de las necesidades de los supervivientes [Internet]. 2012. [Acceso 1 de enero de 2015]. Disponible en: http:// www.gepac.es/docs/informe_supervivientes.pdf

6. Sociedad Española de Oncología Médica SEOM. Largos Supervivientes en Cáncer I. 2012 p. 1-84.

7. Sociedad Española de Oncología Médica SEOM. Largos Supervivientes en Cáncer II. 2012 p. 1-62.

8. Arrighi E, Jovell AJ, Navarro MD, Font A. Desarrollo del cuestionario español para medir necesidades no cubiertas de supervivientes de cáncer (CESC). Psicooncología 2014;11:117-28. DOI: 10.5209/ rev_PSIC.2014.v11.n1.44921

9. Rodríguez E, Font A. Eficacia de la terapia de grupo en cáncer de mama: evolución de las emociones desadaptativas. Psicooncología 2013;10:275-87. Doi: 10.5209/ rev_PSIC.2013.v10.n2-3.43449

10. Rodríguez E, Font A. Eficacia de la terapia grupal en la calidad de vida y el estado emocional en mujeres con cáncer de mama. Psicooncología 201310:275-87. DOI: 10.5209/rev_PSIC.2013.v10.n23.43449

11. Kern de Castro E, Moro L. Factores psicosociales relacionados con el autocuidado en la prevención, tratamiento y postra- 
tamiento del cáncer de mama. Psicooncología 2012;9:453-65. Doi: 10.5209/ rev_PSIC.2013.v9.n2-3.40916

12. Narváez A, Rubiños C, Gómez R, García A, Cortés-Funes F. Valoración de la eficacia de una terapia grupal cognitivoconductual en la imagen corporal, autoestima, sexualidad y malestar emocional (ansiedad y depresión) en pacientes de cáncer de mama. Psicooncología 2008;5:93-102.

13. Blázquez A, Nierga J, Javierre C. Terapia a través de la danza y el movimiento en mujeres con cáncer de mama: el impacto psicofísico del proceso empático. Psicooncología. 2011; 8:31-43. Doi:10.5209/rev_ PSIC.2011.v8.n1.3

14. Carballo P, Martí P. Musicoterapia y Oncología: Una experiencia con un grupo de mujeres con cáncer de mama. Música Ter Comun 2008;28:27-42.

15. Collette N. Arteterapia y cáncer. Psicooncología 2011;8:81-99. Doi: 10.5209/rev_ PSIC.2011.v8.n1.7

16. Martí P, Carballo P. Musicoterapia y cáncer de mama. En: Martí P, Mercadal-Brotons $\mathrm{M}$, editores. Musicoterapia en Medicina Aplicaciones prácticas. Barcelona: Editorial Médica Jims.; 2010. p. 251-70.

17. WFMT. World Federation for Music Therapy WFMT [Internet]. What is music therapy? 2011 [Acceso 10 de enero de 2015]. Disponible en: http://www.wfmt.info/ wfmt-new-home/about-wfmt/

18. American Music Therapy Association (AMTA) [Internet]. Definition and quotes about music therapy [Internet]. [Acceso 26 de febrero de 2015]. Disponible en: http:// www.musictherapy.org/about/quotes/

19. Alvin J. Musicoterapia. Barcelona: Paidós; 1984

20. Maranto CD. A classification model for Music and Medicine. Applications of Music in Medicine. Washington, DC: National Association for Music Therapy,1991.p.1-6.

21. Dileo C. Effects of music and music therapy on medical patients: a meta-analysis of the research and implications for the future. J Soc Integr Oncol 2006;4:67-70. Doi: 10.2310/7200.2006.002

22. Standley JM. Investigación sobre música en el tratamiento médico. En: Martí $\mathrm{P}$, Mercadal-Brotons $M$, editores. Musicoterapia en medicina aplicaciones prácticas. Barcelona: Editorial Médica Jims.; 2010. p. 1-63.

23. Munro S, Mount B. Music therapy in palliative care. Can Med Assoc J 1978;119:102934.

24. O'Callaghan C, Hiscock R. Interpretive subgroup analysis extends modified grounded theory research findings in oncologic music therapy. J Music Ther 2007;44:256-81. Doi: 10.1093/jmt/44.3.256

25. Magill L. Role of music therapy in integrative oncology. J Soc Integr Oncol 2006;4:7981. Doi: 10.2310/7200.2006.009

26. Aldridge D. Music Therapy research in the medical literature. Music therapy research and practice in medicine from out of silence. London: Jessica Kingsley Publishers, 1996.p 59-84.

27. Bellamy MA, Willard PB. Music Therapy: An integral component of the oncology experience. Int J Arts Med 1993;II:14-9.

28. Hilliard RE. The use of music therapy in meeting the multidimensional needs of hospice patients and families. J Palliat Care 2001;17:161-6.

29. Kruse J. Music therapy in United States cancer settings: Recent trends in practice. Music Ther Perspect 2003;21:89-98. Doi:10.1093/mtp/21.2.89

30. Mandel SE, Davis BA, Secic M. Effects of music therapy on patient satisfaction and health-related quality of life of hospital inpatients. Hosp Top 2014;92:28-35. Doi: 10.1080/00185868.2014.906830.

31. O'Brien EK. Cancer patients' evaluation of a music therapy program in a public adult hospital. En: Rebollo Pratt R, Grocke DE, editors. Music Medicine 3. Parkville. Victoria: University of Melbourne,1999.p. 285-300. 
32. O'Kelly J, Koffman J. Multidisciplinary perspectives of music therapy in adult palliative care. Palliat Med 2007;21:23541. Doi:10.1177/0269216307077207

33. Porchet-Munro S. Music therapy perspectives in palliative care education. J Palliat Care 1993;9:39-42.

34. Hanser S. Music therapy in adult oncology: Research issues. J Soc Integr Oncol 2006;4:62-6.

35. Domar AD, Eyvazzadeh A, Allen S, Roman K, Wolf R, Orav J, et al. Relaxation techniques for reducing pain and anxiety during screening mammography. AJR Am J Roentgenol 2005;184:445-7. Doi: 10.2214/ajr.184.2.01840445

36. Haun M, Mainous RO, Looney SW. Effect of music on anxiety of women awaiting breast biopsy. Behav Med 2001;27:12732. Doi:10.1080/08964280109595779

37. Zhou K, Li X, Yan H, Dang S, Wang D. Effects of music therapy on depression and duration of hospital stay of breast cancer patients after radical mastectomy. Chin Med J (Engl) 2011;124:2321-7. Doi:10.1016/j.ejon.2014.07.010

38. Li X-M, Yan H, Zhou K-N, Dang S-N, Wang D-L, Zhang Y-P. Effects of music therapy on pain among female breast cancer patients after radical mastectomy: Results from a randomized controlled trial. Breast Cancer Res Treat 2011;128:411-9. Doi:10.1007/ s10549-011-1533-z

39. Li X, Zhou K, Yan H, Wang D, Zhang Y. Effects of music therapy on anxiety of patients with breast cancer after radical mastectomy: A randomized clinical trial. J Adv Nurs. 2012;68:1145-55. Doi:0.1111/ j.1365-2648.2011.05824.x

40. Bulfone T, Quattrin R, Zanotti R, Regattin L, Brusaferro S. Effectiveness of music therapy for anxiety reduction in women with breast cancer in chemotherapy treatment. Holist Nurs Pract 2009;23:238-42. Doi:10.1097/HNP.0b013e3181aeceee.

41. Ferrer AJ. The effect of live music on decreasing anxiety in patients undergoing chemotherapy treatment. J Music Ther 2007;44:242-55. Doi:10.1093/jmt/44.3.242

42. Frank JM. The effects of music therapy and guided visual imagery on chemotherapy induced nausea and vomiting. Oncol Nurs Forum 1985;12:47-52.

43. Gimeno MM. El uso de la música y la imagen en oncología. En: Marti P, MercadalBrotons M, editores. Musicoterapia en medicina aplicaciones prácticas. Barcelona: Editorial Médica Jims, 2010.p.237-50.

44. Good M, Picot BL, Salem SG, Chin CC, Picot SF, Lane D. Cultural differences in music chosen for pain relief. J Holist Nurs 2000;18:245-60. Doi:10.1177/089801010001800306

45. Sabo CE, Michael SR. The influence of personal message with music on anxiety and side effects associated with chemotherapy. Cancer Nurs 1996;9:283-9. Doi:10.1097/00002820-19960800000004

46. Standley JM. Clinical application of music and chemotherapy: The effects on nausea and emesis. Music Ther Perspect 1992;10:27-35. Doi:10.1093/mtp/10.1.27

47. Tilch S, Haffa-Schmidt $U$, Wandt $H$, Kappauf H, Schafer K, Birkmann J. Supportive music therapy improves mood states in patients undergoing myeloblative chemotherapy. Bone Marrow Transpl 1999;23(Suppl.1):566.

48. Bozcuk H, Artac M, Kara A, Ozdogan M, Sualp Y, Topcu Z, et al. Does music exposure during chemotherapy improve quality of life in early breast cancer patients? A pilot study. Med Sci Monit 2006;12:CR200-5.

49. Serra Vila M. Comparación del efecto de una intervención con musicoterapia para mujeres con cáncer de mama durante la sesión de quimioterapia: un análisis cuantitativo y cualitativo [Internet]. [Acceso 26 de febrero de 2015]. [Barcelona]: Universitat Ramon Llull; 2013. Disponible en: http://www.tdx.cat/bitstream/handle/10803/108962/TESIS\%20MIREIA\%20 SERRA\%20VILA_URL.pdf?sequence $=3$ 
50. O'Callaghan C, Sproston M, Wilkinson K, Willis D, Milner A, Grocke D, et al. Effect of self-selected music on adults' anxiety and subjective experiences during initial radiotherapy treatment: $\mathrm{A}$ randomised controlled trial and qualitative research. J Med Imaging Radiat Oncol 2012;56:473-7. Doi: 10.1111/j.1754-9485.2012.02395.x

51. Clark M, Isaacks-Downton G, Wells N, Redlin-Frazier S, Eck C, Hepworth JT, et al. Use of preferred music to reduce emotional distress and symptom activity during radiation therapy. J Music Ther 2006;43:24765. Doi:10.1093/jmt/43.3.247

52. Smith $M$, Casey $L$, Johnson $D$, Gwede C, Riggin OZ. Music as a therapeutic intervention for anxiety in patients receiving radiation therapy. Oncol Nurs Forum 2001;28:855-62.

53. Chuang C-Y, Han W-R, Li P-C, Song M-Y, Young S-T. Effect of long-term music therapy intervention on autonomic function in anthracycline-treated breast cancer patients. Integr Cancer Ther $2011 ; 10: 312-6$. Doi: $10.1177 / 1534735411400311$.

54. Beggs C. Life review with a palliative care patient. Case studies in Music Therapy. Phoenixville, PA: Barcelona Publishers; 1991. p. 611-6.

55. Curtis S. The effects of music on pain relief and relaxation of the terminally ill. J Music Ther. 1986;23:10-24. Doi:10.1093/ jmt/23.1.10

56. Hilliard RE. The effects of music therapy on the quality and length of life of people diagnosed with terminal cancer. J Music Ther ;40:113-37. Doi:10.1093/ jmt/40.2.113

57. Hogan BE. The experience of music therapy for terminally ill patients; a phenomenological research project. En: Rebollo Pratt R, Erdonmez-Grocke D, editores. Music Medicine. Melbourne: University of Melbourne, 1999. p.242-52.

58. Magill L. The use of music therapy to address the suffering in advanced cancer pain. J Palliat Care 2001;17:167-72.
59. Mandel SE. The role of the music therapist on the hospice/palliative care team. J Palliat Care 1993;9:37-9.

60. Martin JA. Martin JA. Music therapy at the end of life. En: Bruscia KE, editor. Case studies in Music Therapy. Phoenixville, PA: Barcelona Publishers, 1991. p. 617-32.

61. Mramor KM. Music therapy with persons who are indigent and terminally ill. J Palliat Care; 2001;17:182-7.

62. Nakayama H, Kikuta F, Takeda H. A pilot study on effectiveness of music therapy in hospice in Japan. J Music Ther 2009;46:16072. Doi:10.1093/jmt/46.2.160

63. O'Kelly J. Music therapy in palliative care: Current perspectives. Int J Palliat Nurs 2002;8:130-6. Doi:10.12968/ ijpn.2002.8.3.10249

64. Salmon D. Music therapy as psychospiritual process in palliative care. J Palliat Care 2001;17:142-6.

65. West T. Psychological issues in hospice music therapy. Music Ther Perspect 1994;12:125-9. Doi: 10.1093/ $\mathrm{mtp} / 12.2 .117$

66. Whitall J. Whitall, J. En: Brurscia KE, editor. Case studies in Music Therapy. Phoenixville, PA: Barcelona Publishers; 1991.p.603-10.

67. Wlodarczyk N. The effect of music therapy on the spirituality of persons in an in-patient hospice unit as measured by self-report. J Music Ther 2007;44:113-22. Doi:10.1093/jmt/44.2.113

68. Allen JL. The effectiveness of group music psychotherapy in improving the selfconcept of breast cancer survivors. 2010. University of Temple. [Internet]. [Acceso 10 de febrero de 2015] Disponible en: http://digital.library.temple.edu/cdm/ref/ collection/p245801coll10/id/104147

69. Conradi Stark J. Perceived benefits of group music therapy for breast cancer survivors: mood, psychosocial wellbeing, and quality of life. Michigan State University; 2012. [Internet]. [Acceso 10 de febrero de 2015] Disponible 
en: http://ir.uiowa.edu/cgi/viewcontent. cgi?article $=2602 \&$ context $=$ etd

70. Stordahl JJ. The influence of music on depression, affect, and benefit finding among women at the completion of treatment for breast cancer [Internet]. ProQuest Dissertations and Theses. University of Miami; 2009. [Internet]. [Acceso 10 de febrero de 2015] Disponible en: http://scholarlyrepository.miami.edu/cgi/viewcontent. cgi ?article $=1336 \&$ context $=$ oa_dissertations

71. Burns DS. The effect of the bonny method of guided imagery and music on the mood and life quality of cancer patients. J Music Ther 2001;38:51-65. Doi:10.1093/ jmt/38.1.51

72. Burns DS, Sledge RB, Fuller LA, Daggy JK, Monahan PO. Cancer patients' interest and preferences for music therapy. J Music Ther 2005;42:185-99. Doi:10.1093/ jmt/42.3.185

73. Cassileth BR, Vickers AJ, Magill LA. Music therapy for mood disturbance during hospitalization for autologous stem cell transplantation: A randomized controlled trial. Cancer 2003;98:2723-9. Doi: 10.1002/ cncr.11842

74. Krout RE. The effects of single-session music therapy interventions on the observed and self-reported levels of pain control, physical comfort, and relaxation of hospice patients. Am J Hosp Palliat Care 2001;18:383-90. Doi:10.1177/104990910101800607

75. McDougal Miller D. The effect of music therapy on the immune and adrenocortical systems of cancer patients. University of Kansas, Lawrence.; 1992.

76. Martí P, Mercadal-Brotons M. Musicoterapia en Medicina. Aplicaciones prácticas. Barcelona: Editorial Médica Jims.; 2010.

77. Zigmond AS, Snaith RP. The hospital anxiety and depression scale. Acta Psychiatr Scand 1983;67:361-70. Doi:10.1111/j.1600-0447.1983.tb09716.x

78. McNair DM, Lorr M, Droppelman LF. ED-
ITS manual for the Profile of Mood States. San Diego, CA: Educational \& Industrial Testing Service; 1992.

79. Aaronson NK, Ahmedzai S, Bergman B, Bullinger M, Cull A, Duez NJ, et al. The European Organization for Research and Treatment of Cancer QLQ-C30: A qualityof-life instrument for use in international clinical trials in oncology. J Natl Cancer Inst 1993;85:365-76. Doi:10.1093/ jnci/85.5.365

80. Caro I, Ibáñez E. La Escala Hospitalaria de Ansiedad y Depresión. Su utilidad práctica en Psicología de la Salud. Bol Psicol 1992;36:43-69.

81. Fuentes Y, García Merita M, Melià JL, Balaguer Y. Formas paralelas de la adaptación valenciana del Pefil de Estados de Ánimo (POMS). IV Congreso de Evaluación Psicológica, 21-24 de Septiembre. Santiago de Compostela. España; 1994.

82. Arrarás JJ, Arias de la Vega F, Illarramendi JJ, Manterola A, Salgado E, Domínguez MA, et al. Calidad de vida relacionada con la salud en los servicios de Oncología del Hospital de Navarra. Grupo de Calidad de Vida de la EORTC. An Sist Sanit Navarra 2011;34:9-20. Doi:10.4321/ S1137-66272011000100002

83. Waldon EG. The effects of group music therapy on mood states and cohesiveness in adult oncology patients. J Music Ther 2001;38:212-38. Doi:10.1093/ jmt/38.3.212

84. Bradt J, Dileo C, Grocke D, Magill L. Music interventions for improving psychological and physical outcomes in cancer patients ( Review ). Cochrane database Syst Rev 2011;8. Art. No.: CD006911. Doi: 10.1002/14651858.CD006911.pub2.

85. Dvorak A. Music therapy support groups for cancer patients and caregivers [Internet]. University of Iowa; 2011. [Internet]. [Acceso 1 de febrero de 2015] Disponible en: http://ir.uiowa.edu/cgi/viewcontent. cgi ?article $=2602 \&$ context $=$ etd \&unstamp $\mathrm{ed}=1$ 
86. Huang S-T, Good M, Zauszniewski JA. The effectiveness of music in relieving pain in cancer patients: A randomized controlled trial. Int J Nurs Stud 2010; 47:1354-62. Doi: 10.1016/j.ijnurstu.2010.03.008

87. Magill-Levreault L. Music therapy in pain and symptom management. J Palliat Care 1993;9:42-8.

88. Zimmerman L, Pozehl B, Duncan K, Schmitz R. Effects of music in patients who had chronic cancer pain. West J Nurs Res 1989;11:298-309. Doi:10.1177/019394598901100304
89. Gimeno MM. The effect of music and imagery to induce relaxation and reduce nausea and emesis in cancer patients undergoing chemotherapy treatment. Music Med 2010;2:174-81, Doi:10.1177/1943862110374622

90. Hanser SB, Bauer-Wu S, Kubicek L, Healey M, Manola J, Hernandez M, et al. Effects of a music therapy intervention on quality of life and distress in women with metastatic breast cancer. J Soc Integr Oncol;2006;4:116-24. Doi:10.2310/7200.2006.014 